\title{
Alcohol Consumption and Cardiovascular Disease Risk: Placing New Data in Context
}

\author{
Anna G. Hoek ${ }^{1} \cdot$ Sabine van Oort ${ }^{1} \cdot$ Kenneth J. Mukamal ${ }^{2} \cdot$ Joline W. J. Beulens $^{1,3}$
}

Accepted: 22 October 2021 / Published online: 7 February 2022

(c) The Author(s) 2022

\begin{abstract}
Purpose of Review A clear link between excessive alcohol consumption and cardiovascular disease (CVD) has been established, but no consensus exists on the effects of moderate alcohol consumption on CVD.

Recent Findings A lower risk of coronary heart disease and myocardial infarction among moderate drinkers compared to abstainers has been consistently observed in epidemiological studies and meta-analyses of these studies. However, ambiguity remains on the effect of alcohol on other CVDs and all-cause mortality. Short-term randomized controlled trials (RCT) have identified potentially beneficial effects of alcohol consumption on cardiovascular risk factors, but studies investigating genetic polymorphisms that influence alcohol consumption (i.e., Mendelian randomization) have yielded inconclusive results. To date, a long-term RCT providing causal evidence is lacking but urgently needed.

Summary Triangulation of evidence from different study designs, including long-term RCTs, pragmatic trials and the evaluation of policy measures, combined will lead to the best available evidence.
\end{abstract}

Keywords Alcohol consumption $\cdot$ Cardiovascular disease $\cdot$ Moderate drinking

\section{Introduction}

This article is part of the Topical Collection on Nutrition

Anna G. Hoek

a.g.hoek@amsterdamumc.nl

Sabine van Oort

s.vanoort1@amsterdamumc.nl

Kenneth J. Mukamal

kmukamal@bidmc.harvard.edu

Joline W. J. Beulens

j.beulens@amsterdamumc.nl

1 Amsterdam UMC, Vrije Universiteit Amsterdam, Department of Epidemiology \& Data Science, Amsterdam Cardiovascular Sciences Research Institute, De Boelelaan 1117, Amsterdam, The Netherlands

2 Beth Israel Deaconess Medical Center, Harvard Medical School and Harvard TH Chan School of Public Health, Boston, MA, USA

3 University Medical Centre Utrecht, Julius Center for Health Sciences and Primary Care, Utrecht University, Utrecht, The Netherlands
Alcohol consumption has been extensively studied as a modifiable risk factor for cardiovascular diseases (CVD). Excessive alcohol consumption ( $>60 \mathrm{~g} /$ day in men and $>40 \mathrm{~g} /$ day in women) [1] is a well-known contributor to mortality and burden of CVDs [2, 3]. In contrast, a large number of observational studies report beneficial associations of low to moderate alcohol consumption (up to $60 \mathrm{~g} / \mathrm{day}$ in men and up to $40 \mathrm{~g} /$ day in women) [1] with CVD. This results in a characteristic biphasic, "J-shaped" risk profile $[4,5 \bullet \bullet, 6]$ in which for low to moderate alcohol consumption, a lower CVD risk is observed compared to abstaining and excessive drinking. However, since most of the evidence of the protective effects of low to moderate alcohol consumption on CVD originates from observational studies, the question remains whether this effect is truly causal or merely a result of different forms of bias inherent to observational study designs $[5 \bullet \bullet, 6]$. To answer this question, there is an urgent need for studies assessing causality in the relationship between light to moderate alcohol consumption and CVDs. Unfortunately, to date, large-scale randomized controlled trials (RCT) with a sufficient follow-up period are lacking and previous efforts to conduct such studies have faced administrative and 
political challenges, despite intriguing evidence of feasibility [7-9].

Given the lack of an RCT that can serve as a gold standard, the focus in research has now shifted to new analytical methods and epidemiological designs such as pooled analyses from large consortia, instrumental variable analyses using genetic polymorphisms (e.g., Mendelian randomization (MR) studies), and RCTs investigating intermediate endpoints in the hope of shedding new light on the association between alcohol consumption and CVD. However, none of these studies has been able to resolve the pressing question of whether there is a true protective effect of moderate alcohol consumption. In addition, while such studies are innovative and potentially informative, they are not free of their own limitations and caution is equally required when interpreting the results of these new studies.

Our review aims to summarize previous efforts to investigate the relationship of alcohol consumption with CVD risk using classic observational epidemiologic designs, RCTs and MR studies. We will elaborate on the strengths and weaknesses of the different designs and offer new directions for research for the future.

\section{Evidence from Observational Research}

Over the last five decades [10], hundreds and perhaps thousands of observational studies, systematic reviews and combined meta-analyses investigating the associations of alcohol consumption with CVD and mortality have been published. Observational studies have consistently but not invariably found that alcohol consumption above recommended limits is associated with a higher CVD incidence, cardiovascular and total mortality [2,3]. However, more variable associations have been reported in studies investigating the impact of alcohol consumption within recommended limits on CVD incidence and mortality, compared to alcohol abstainers and excessive drinkers.

Early studies investigating alcohol consumption and cardiovascular health outcomes observed a "J"-shaped association, indicating a reduced risk for CVD incidence, specific and all-cause mortality for within-guideline drinkers compared to either abstainers or excessive drinkers. Protective associations have been best documented for myocardial infarction [11-14], but are also found when investigating heart failure [15, 16], peripheral artery disease [15], abdominal aortic aneurysm [15], hypertension [14], type 2 diabetes [17-19], (ischemic) stroke [14, 15, 20], cardiovascular mortality $[15,21]$ and all-cause mortality $[12,14]$. Furthermore, these lower risks have been corroborated by meta-analyses $[3,4,16,22,23]$. Recently, several large studies and metaanalyses that used updated methodology, with the aim of minimizing the impact of bias inherent to observational study designs, have challenged the apparent association of limited alcohol consumption with lower cardiovascular risk [5••, 6, 24-26]. In a meta-analysis, Wood et al. analyzed data of 599,912 current drinkers without baseline CVD and found linear direct associations between alcohol consumption and risks of stroke, coronary disease, heart failure, fatal hypertensive disease and fatal aortic aneurysm, and inverse associations with overall cardiovascular disease and myocardial infarction for intake below $200 \mathrm{gm} /$ week [5••]. A second meta-analysis executed by the Global Burden of Diseases, Injuries and Risk Factors Study (GBD) found similar results [6]. In both meta-analyses, no harmful or beneficial association of limited drinking with all-cause mortality was found, although Wood et al. observed lower risk among consumers of $<300 \mathrm{gm} /$ week whose consumption was distributed over 3 or more days per week (i.e., within guidelines) $[5 \bullet \bullet, 6]$. The authors argue that the general assumption of a protective association of limited alcohol intake on CVD is flawed, and conclude that the relationship between alcohol consumption and CVD risk is complex and does not express itself as a single $\mathrm{J}$-shaped association [5••]. Even though these studies consist of larger sample sizes and use updated methodology, they are not immune to selection bias and confounding inherent to observational research. Furthermore, combining multiple datasets into one mega cohort could actually cause data loss, therefore worsening these distorting factors and making outcomes less generalizable [27].

Even more recent observational studies have focused on specific niches of the relationship between alcohol consumption and CVD, testing the hypothesis that the relationship between alcohol and CVD does not fit into a one-size-fitsall approach and that the conflicting results found in previous studies might be due to differences within subpopulations. For example, investigators have observed apparent differences in the alcohol-CVD relationship between African-Americans, Asian-Americans and other race/ethnicity groups found [28-32] (perhaps related to well-known genetic differences in alcohol metabolism [33, 34]). Other recent papers have addressed drinking patterns, suggesting that regular drinking and drinking with meals may be associated with a lower risk of mortality [35-37] and beverage types, arguing for unique anti-oxidative and antiinflammatory effects of red wine consumption [38]. However, although differences in subpopulations are present and should be taken into account in future guidelines, these studies have not resolved the question whether limited alcohol consumption is protective or harmful for the development of CVD. 


\section{Methodological Problems Arising from Observational Research}

When interpreting results from observational studies, several forms of bias should be considered. The first factor is the presence of uncontrolled confounding [39]. It is nearly impossible to account for all confounding factors in observational study designs, and this is likely to be particularly true for alcohol consumption, which has strong and varied determinants of exposure [25, 39-41]. A second caveat to consider is the "sick quitter" phenomenon, whereby abstainers (the referent category in many studies) include a mixture of long-term abstainers and those who have quit due to preexisting illness. This results in an artificial elevation of the health risk among abstainers, in which it is not the absence of alcohol but impaired health status that increases the observed elevated risk [25, 41-50]. Even though the sickquitter phenomenon can be partly solved by careful separation of abstainers category into former and never drinkers or by using rare alcohol intake as a reference category in analyses, it is hard to fully account for, and the use of rare drinkers poses its own problems due to underestimation.

A third problem in observational research is the use of questionnaires as a self-reported measurement to assess alcohol consumption, which is prone to systematic error due to recall bias [51, 52], underreporting [53], misreporting [53-55] and adherence to social and cultural norms in answering the questions $[56,57]$. This can theoretically be overcome by using biomarkers of alcohol consumption as tool to assess habitual alcohol consumption [58]. However, most biomarkers for alcohol consumption are either not specific enough (e.g. liver function markers) or only capture recent consumption of the past days, often at high doses (e.g., ethyl glucuronide). Finally, the lack in uniformity on the definition of alcohol intake categories, the definition of abstinence and changes in drinking pattern over time are not standardized, resulting in heterogeneity of alcohol-related measurements, which makes it difficult to combine multiple datasets into larger analyses [53].

Although these forms of bias are inherent to observational studies, they do not diminish the importance of such studies to identify potential associations and they remain the only available approach to date to directly link alcohol consumption with long-term outcomes.

\section{Evidence from Randomized Controlled Trials}

RCTs are not influenced by these forms of bias and are often regarded as the gold standard to prove causality in the relations between a risk factor and outcome. Due to the difficulty and costs of performing a long-term RCT of limited alcohol consumption on hard outcomes such as CVD, the main body of evidence comes from short-term RCTs on cardiovascular risk factors. Several meta-analyses show that moderate alcohol consumption increased high-density lipoprotein (HDL) cholesterol, apolipoprotein A1 and adiponectin [59•, 60, 61]. Moderate alcohol consumption has also shown to reduce low-density lipoprotein cholesterol, fibrinogen levels, Interleukin-6, HbA1c and fasting insulin concentrations in various studies [59•, 61]. No effects were observed on C-reactive protein or total cholesterol [59•, 61]. A meta-analysis of trials investigating the effect of a change of alcohol consumption on blood pressure showed that a reduction of alcohol consumption in those who drank two drinks/day or less was not effective, while this resulted in a significant reduction in systolic and diastolic blood pressure for heavier drinkers [62]. A meta-analysis of RCTs up to 2017 performed in people with diabetes showed no effect of moderate alcohol consumption during 4 to 104 weeks on HbA1c or blood glucose [63], although the longest such study to date identified a benefit specifically among those with slow ethanol metabolism [8]. Altogether, these studies provide plausible underlying mechanisms not only for the observed risk reduction of myocardial infarction with moderate alcohol consumption, but also for increased risks of other cardiovascular outcomes such as heart failure or stroke.

Despite the plausibility of this evidence, the duration of most RCTs has been 4 to 8 weeks, and effects over longer time periods on hard outcomes are therefore uncertain. Only a handful of RCTs have been performed with a duration of over 1 year. These RCTs, mainly conducted in high-risk populations with diabetes or previous myocardial infarction, showed improvements of HDL cholesterol, inflammatory markers and insulin sensitivity and even echocardiographically determined left ventricular function [9, 64], generally in line with shorter trials. The meta-analysis of RCTs on reduction of alcohol consumption on blood pressure included four trials with a duration of 1 year or longer, and these results were in line with the overall meta-analysis [62]. These longer trials suggest feasibility of a longer term RCT on hard outcomes to resolve the ongoing debate about effects of light to moderate alcohol consumption on CVD. Despite this, a recent effort to conduct such a long-term trial-the Moderate Alcohol and Cardiovascular Health trial (MACH15) - seemed feasible, but was terminated prematurely by the US National Institutes of Health, which funded the trial [7, 65]. 


\section{Executional Problems Regarding Randomized Controlled Trials}

In contrast to the methodological problems faced when conducting and interpreting results from observational studies, important practical and ethical concerns face large-scale, longterm RCTs [7, 65]. Besides the question whether it is justifiable to impose alcohol consumption on individuals-although MACH15 was designed to exclude abstainers and heavy drinkers-one of the major concerns is on how the general public will conceive possible outcomes of RCTs. Since RCTs require strict inclusion and exclusion criteria, the results are by definition definitively applicable only to a selected part of the population. Because alcoholic beverages are widely consumed worldwide, the danger is that results of an RCT executed in a specific population could be wrongfully be applied to excluded individuals in whom the results would have differed [65]. Furthermore, several potentially adverse but rare outcomes, such as the risk of developing specific types of cancer, are impossible to investigate in an RCT due to the unrealistically large sample sizes needed. To date, all attempts to execute a largescale RCT with sufficient follow-up time have stranded and currently no large RCTs are running to our knowledge.

\section{Evidence from Mendelian Randomization Studies}

Facilitated by the availability of large genome-wide association studies (GWAS), MR has been increasingly used to investigate the relationship between alcohol consumption and CVD [66, 67•]. MR is a genetic instrumental variable analysis that uses genetic variants that are robustly associated with modifiable risk factors as instrumental variables [68]. Since genetic variants are randomly allocated at meiosis, they mimic an RCT setting in which all other variables except the exposure are distributed equally between subgroups. This theoretically makes the design less vulnerable to confounding and reverse causation bias [68], provided all instrumental variable assumptions hold.

For alcohol consumption and hard CVD endpoints, the majority of the MR studies thus far have reported null associations, but some have reported a higher risk of ischemic heart disease, peripheral artery disease, stroke and diabetes [67•]. For intermediate endpoints, evidence from MR studies has been more consistent, with the majority of the studies showing that genetically predicted higher alcohol consumption was associated with higher levels of blood pressure, triglycerides, HDL cholesterol, glucose and body mass index, and with lower levels of low-density lipoprotein cholesterol [67•]. The majority of methods used in MR assume linearity in the alcohol-outcome relationship, and are therefore not suitable to answer questions on relationships that are potentially non-linear, as could be the case with alcohol and CVD. Recently, new methodology has been developed to study potential non-linearity, such as the localized average causal effects (LACE) method [69], but its validity remains uncertain and, to date, only a few studies have used this method, with inconsistent results on the shape of the association [70, 71].

\section{Limitations of Using Mendelian Randomization Studies in Alcohol Research}

MR studies allow for assessing causality when all assumptions are met, meaning the genetic variant (1) is robustly associated with the exposure, (2) is not associated with any confounder of the exposure outcome association and (3) only affects the outcome via its association with the exposure [27, 68, 72]. However, the use of SNPs related to alcohol consumption inherently poses a threat to these assumptions. Firstly, the explained variance for genetic instruments for alcohol consumption is generally low; therefore, large samples sizes are needed [67•]. Secondly, genetic instruments for alcohol consumption related to functional genes (e.g. ALDH2 and ADH1B/C) can be used in MR studies and explain a large part of the variance in Asian populations, but explain only a small part of the variance of alcohol consumption in non-Asian populations. Therefore, in non-Asian populations, other variants that are significantly associated with alcohol consumption, discovered through GWAS analyses, are commonly used. However, since the causality of these additional genes is not defined, it is difficult to assess whether the exposure gene is not associated with any confounder of the exposure-outcome association therefore possibly violating the second assumption [67•]. Thirdly, the most commonly used genetic variants for alcohol consumption share a similar pathway with variants associated with problematic alcohol consumption; therefore, the presence of pleiotropy is hard to avoid [73].

Furthermore, a few additional limitations are of special importance when using MR to investigate the role of alcohol consumption. Firstly, as mentioned above, to date, investigating potential non-linearity is difficult in such analyses and often not carried out. Furthermore, bias can be introduced in MR studies via assortative mating and dynastic effects [74, 75]. Assortative mating occurs if an individual with a particular genetic predisposition bases his partner selection on a certain genetically influenced phenotype. Dynastic effects are similar and occur when the expression of the parental genotype in the parental phenotype has a direct effect on their offspring's phenotype. These are environmental and social factors that have the potential to affect the distribution of genetic variants for 
specific traits within the population. Studies of other exposures (e.g., BMI or education) have indeed shown that this leads to bias by inducing an association between the instrumental variable and the outcome $[74,75]$. Although this has not been investigated for alcohol consumption, an earlier study provided evidence for presence of assortative mating for alcohol consumption [75], and parental substance use disorder is known to influence adult chronic diseases [76], suggesting dynastic effects. Finally, no evidence currently exists that genetic variants can separate the various domains of alcohol consumption (e.g., quantity versus frequency), despite their vastly different associations with CVD. Altogether, MR studies provide evidence from a different angle, but can by themselves not solve the debate on the role of limited drinking on cardiovascular health.

\section{A Century of Research on the Relationship of Alcohol and Cardiovascular Disease and Still No Consensus: How Do We Move Forward?}

After nearly a century of research on the effects of alcohol consumption on cardiovascular health, we find ourselves running in circles, asking the same questions and reporting the same limitations. To move forward and gain greater insight into the health effects of limited alcohol consumption, a reconsideration of the standard of evidence is needed. Despite novel techniques to assess causality, a large-scale, long-term RCT still seems to be the only option to resolve the debate.

The design and initial conduct of the MACH15 trial show the feasibility of executing a large-scale trial. However, the conduct of such a trial itself became a matter of debate [65]. Although a trial of limited alcohol consumption could potentially show effects on CVD when using a high-risk population, it is unlikely that such a trial can definitively quantify effects on some adverse events such as breast cancer, simply because they are too rare (fortunately) and thus require astronomical sample sizes. Sample size calculations show that 60,000 individuals are needed to detect the expected risk of any alcohol-related cancer, and when aiming to investigate specific forms of cancer, such as breast cancer, up to five times bigger samples are needed [7]. Since in observational studies, limited alcohol consumption has no beneficial association with most cancers, a RCT specifically to prove alcohol causes cancer is ethically dubious. Secondly, the fear of falsely extrapolating results from a specific and high-risk study population to a more general public has been expressed. However, we argue that if a protective effect is observed in a high-risk population, these effects are likely to be physiologically generalizable to a lower risk population, albeit with a smaller absolute risk reduction. Most importantly, the fear of misinterpretation should not be a reason not to execute a RCT, but rather should motivate investigators to strive for excellent communication and prevent misinterpretation of the results [7]; fear of misinterpretation would otherwise derail virtually every important trial. Ultimately, we emphasize that alcohol is consumed by half of the world's population, and to date, there is a nearly complete lack of causal evidence on its long-term effects. Therefore, obtaining highest level of evidence-in an appropriate way-is in everyone's benefit. To argue otherwise is to leave patients, physicians and public health professionals in a state of artificially engineered ignorance.

With no current RCTs running, it is likely that some time will pass before gold standard evidence is obtained. So, how do we move forward? The execution of a pragmatic trial, investigating the effects of lowering alcohol consumption on CVD endpoints, might be a solution. A pragmatic trial aims to evaluate whether a treatment works in daily clinical practice by using less controlled settings than when executing a classic RCT, but by still using randomization to compare different care strategies [77, 78]. This overcomes ethical concerns such as whether it is justifiable to impose people to drink alcohol, as in a pragmatic trial, the intervention could be to advice a reduction of the amount of alcohol already consumed. An advantage of this approach is the compatibility with the usual care situation, where a physician advises individuals to lower their alcohol consumption. Although these less controlled settings lead to the situation that the true biological "causal" effect cannot be estimated [79], it would provide more generalizable findings. Furthermore this design lends itself perfectly for stratification of the results in categories of alcohol consumption at baseline, which is insightful given the debate surrounding the possible J-shaped curve in the relationship of alcohol consumption on CVD outcomes. An alternative path to explore is the evaluation of the impact of alcohol consumption policy measures, in which pre- and post-intervention data in an interrupted time series analysis can be compared without using randomization [80,81]. Alcohol consumption might lend itself particularly well for this kind of research, since the ambiguity on the relationship between limited alcohol consumption on health outcomes resulted in a large variety and frequent changes of alcohol consumption guidelines worldwide [82, 83]. Nonetheless, these studies too can be affected by confounding due to secular trends that co-occur with alcohol policy changes. Overall, we believe that the evidence from different approaches and study designs, with each their own strengths and limitations, when combined will result into the best available evidence [84, 85] (Fig. 1). Furthermore, to translate the research evidence to prevention in daily care, research on individual patient characteristics and absolute treatment effects is also needed. This can contribute to a tailored prevention approach for individual patients. 


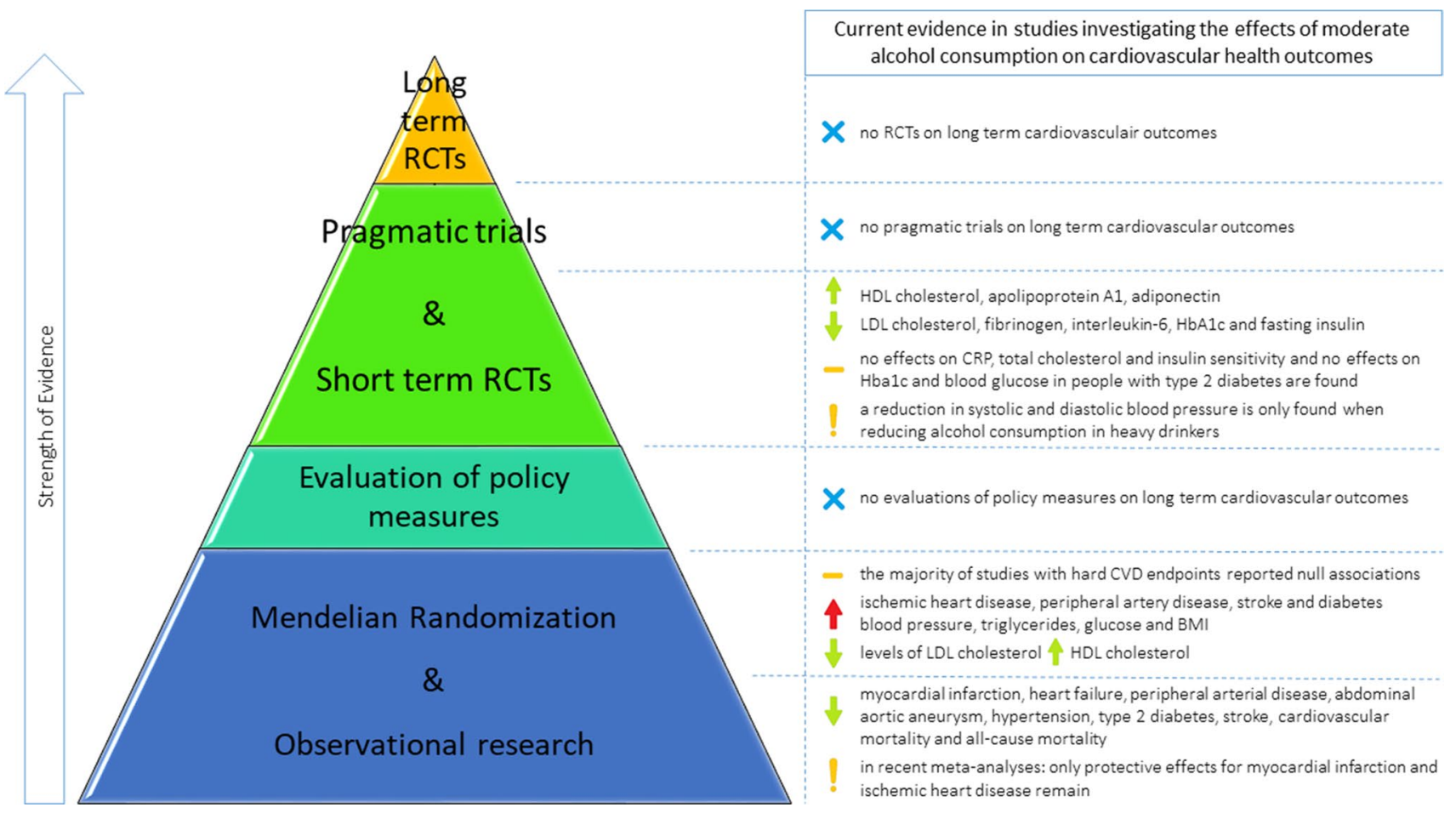

Fig. 1 Overview of the current evidence of moderate alcohol consumption on cardiovascular health outcomes. RCTs: randomized controlled trials; HDL: high-density lipoprotein; LDL: low-density lipoprotein; HbA1C: hemoglobin A1C; BMI: body mass index

One pressing question remains to be answered: is there a safe drinking threshold for moderate amounts of alcohol consumption? Current guidelines generally recommend consumption of 1 drink or less daily ( $14 \mathrm{~g} /$ day) for most individuals; some allow two drinks daily among younger and middle-aged men. In our opinion, these guidelines should be maintained until gold standard evidence is obtained, taking into account individual patient characteristics. And, most importantly, since worldwide alcohol consumption remains alarmingly high with an estimated consumption of 6.41 of pure alcohol per capita [86], promoting adherence to these guidelines should remain a major public health priority.

\section{Conclusion}

Controversy remains regarding the effects of moderate alcohol consumption on CVDs. A lower risk of coronary heart disease and myocardial infarction among moderate drinkers compared to abstainers has been reported in observational studies and was confirmed in the latest meta-analyses. However, on other cardiovascular outcomes and all-cause mortality, conflicting results have been reported. Many short-term RCTs and a few longer term trials have shown potentially beneficial effects of alcohol consumption on cardiovascular risk factors. However, MR studies investigating genetic polymorphisms that influence alcohol consumption often found non-protective effects, although results in MR studies are not always consistent and difficult to generalize (Figure 1).

Since alcohol is consumed by half of the world's population, other approaches should be explored to define a safe limit to alcohol consumption. Future research should focus on executing pragmatic trials investigating the effects of lowering alcohol consumption in a daily clinical practice or on evaluating the impact of certain alcohol consumption regulating policy measures. Even though each of these designs have their own strengths and limitations, combined they can result in a careful triangulation of the evidence.

\section{Compliance with Ethical Standards}

Conflict of Interest Dr. Mukamal reports grants within the last 36 months from the National Institutes of Health, the United States Highbush Blueberry Council, and the American Heart Association and service on a data and safety monitoring board for the University of Pittsburgh for projects unrelated to this manuscript.

Dr. Beulens reports grants from FNIH, outside the submitted work. The other authors declare that they have no conflict of interest.

Human and Animal Rights and Informed Consent This article does not contain any studies with human or animal subjects performed by any of the authors.

Open Access This article is licensed under a Creative Commons Attribution 4.0 International License, which permits use, sharing, 
adaptation, distribution and reproduction in any medium or format, as long as you give appropriate credit to the original author(s) and the source, provide a link to the Creative Commons licence, and indicate if changes were made. The images or other third party material in this article are included in the article's Creative Commons licence, unless indicated otherwise in a credit line to the material. If material is not included in the article's Creative Commons licence and your intended use is not permitted by statutory regulation or exceeds the permitted use, you will need to obtain permission directly from the copyright holder. To view a copy of this licence, visit http://creativecommons. org/licenses/by/4.0/.

\section{References}

Papers of particular interest, published recently, have been highlighted as:

\section{- Of importance}

$\bullet$ Of major importance

1. Fernandez-Sola J. Cardiovascular risks and benefits of moderate and heavy alcohol consumption. Nat Rev Cardiol. 2015;12(10):576-87.

2. Gunzerath L, Faden V, Zakhari S, Warren K. National Institute on Alcohol Abuse and Alcoholism report on moderate drinking. Alcohol Clin Exp Res. 2004;28(6):829-47.

3. Di Castelnuovo A, Costanzo S, Bagnardi V, Donati MB, Iacoviello L, de Gaetano G. Alcohol dosing and total mortality in men and women: an updated meta-analysis of 34 prospective studies. Arch Intern Med. 2006;166(22):2437-45.

4. Ronksley PE, Brien SE, Turner BJ, Mukamal KJ, Ghali WA. Association of alcohol consumption with selected cardiovascular disease outcomes: a systematic review and meta-analysis. BMJ. 2011;342:d671.

5.•• Wood AM, Kaptoge S, Butterworth AS, Willeit P, Warnakula $\mathrm{S}$, Bolton $\mathrm{T}$, et al. Risk thresholds for alcohol consumption: combined analysis of individual-participant data for 599912 current drinkers in 83 prospective studies. Lancet. 2018;391(10129):1513-23. This large meta-analysis failed to find protective effects for moderate alcohol consumption on most cardiovascular outcomes, and the authors argue that the relationship between alcohol consumption and CVD risk is complex and does not express itself as a single $\mathrm{J}$-shaped association.

6. Collaborators GBDA. Alcohol use and burden for 195 countries and territories, 1990-2016: a systematic analysis for the Global Burden of Disease Study 2016. Lancet. 2018;392(10152):1015-35.

7. Spiegelman D, Lovato LC, Khudyakov P, Wilkens TL, Adebamowo CA, Adebamowo SN, et al. The Moderate Alcohol and Cardiovascular Health Trial (MACH15): design and methods for a randomized trial of moderate alcohol consumption and cardiometabolic risk. Eur J Prev Cardiol. 2020;27(18):1967-82.

8. Gepner Y, Golan R, Harman-Boehm I, Henkin Y, Schwarzfuchs $\mathrm{D}$, Shelef I, et al. Effects of initiating moderate alcohol intake on cardiometabolic risk in adults with type 2 diabetes: a 2-year randomized, controlled trial. Ann Intern Med. 2015;163(8):569-79.

9. Marfella R, Cacciapuoti F, Siniscalchi M, Sasso FC, Marchese $\mathrm{F}$, Cinone F, et al. Effect of moderate red wine intake on cardiac prognosis after recent acute myocardial infarction of subjects with Type 2 diabetes mellitus. Diabet Med. 2006;23(9):974-81.
10 Klatsky AL, Friedman GD, Siegelaub AB. Alcohol consumption before myocardial infarction. Results from the Kaiser-Permanente epidemiologic study of myocardial infarction. Ann Intern Med. 1974;81(3):294-301.

11. Mukamal KJ, Conigrave KM, Mittleman MA, Camargo CA Jr, Stampfer MJ, Willett WC, et al. Roles of drinking pattern and type of alcohol consumed in coronary heart disease in men. $\mathrm{N}$ Engl J Med. 2003;348(2):109-18.

12. Britton A, Marmot M. Different measures of alcohol consumption and risk of coronary heart disease and all-cause mortality: 11-year follow-up of the Whitehall II Cohort Study. Addiction. 2004;99(1):109-16.

13. Mukamal KJ, Jensen MK, Gronbaek M, Stampfer MJ, Manson JE, Pischon T, et al. Drinking frequency, mediating biomarkers, and risk of myocardial infarction in women and men. Circulation. 2005;112(10):1406-13.

14. Mostofsky E, Mukamal KJ, Giovannucci EL, Stampfer MJ, Rimm EB. Key findings on alcohol consumption and a variety of health outcomes from the nurses' health study. Am J Public Health. 2016;106(9):1586-91.

15. Bell S, Daskalopoulou M, Rapsomaniki E, George J, Britton A, Bobak M, et al. Association between clinically recorded alcohol consumption and initial presentation of 12 cardiovascular diseases: population based cohort study using linked health records. BMJ. 2017;356:j909.

16. Larsson SC, Wallin A, Wolk A. Alcohol consumption and risk of heart failure: meta-analysis of 13 prospective studies. Clin Nutr. 2018;37(4):1247-51.

17. Howard AA, Arnsten JH, Gourevitch MN. Effect of alcohol consumption on diabetes mellitus: a systematic review. Ann Intern Med. 2004;140(3):211-9.

18. Carlsson S, Hammar N, Grill V. Alcohol consumption and type 2 diabetes Meta-analysis of epidemiological studies indicates a U-shaped relationship. Diabetologia. 2005;48(6):1051-4.

19. Joosten MM, Chiuve SE, Mukamal KJ, Hu FB, Hendriks HF, Rimm EB. Changes in alcohol consumption and subsequent risk of type 2 diabetes in men. Diabetes. 2011;60(1):74-9.

20. Reynolds K, Lewis B, Nolen JD, Kinney GL, Sathya B, He J. Alcohol consumption and risk of stroke: a meta-analysis. JAMA. 2003;289(5):579-88.

21 Mukamal KJ, Chen CM, Rao SR, Breslow RA. Alcohol consumption and cardiovascular mortality among U.S. adults, 1987 to 2002. J Am Coll Cardiol. 2010;55(13):1328-35.

22. Costanzo S, Di Castelnuovo A, Donati MB, Iacoviello L, de Gaetano G. Alcohol consumption and mortality in patients with cardiovascular disease: a meta-analysis. J Am Coll Cardiol. 2010;55(13):1339-47.

23. Xi B, Veeranki SP, Zhao M, Ma C, Yan Y, Mi J. Relationship of alcohol consumption to all-cause, cardiovascular, and cancer-related mortality in U.S. adults. J Am Coll Cardiol. 2017;70(8):913-22.

24. Smyth A, Teo KK, Rangarajan S, O’Donnell M, Zhang X, Rana $\mathrm{P}$, et al. Alcohol consumption and cardiovascular disease, cancer, injury, admission to hospital, and mortality: a prospective cohort study. Lancet. 2015;386(10007):1945-54.

25. Knott CS, Coombs N, Stamatakis E, Biddulph JP. All cause mortality and the case for age specific alcohol consumption guidelines: pooled analyses of up to 10 population based cohorts. BMJ. 2015;350:h384.

26. Stockwell T, Zhao J, Panwar S, Roemer A, Naimi T, Chikritzhs T. Do, "Moderate" drinkers have reduced mortality risk? A systematic review and meta-analysis of alcohol consumption and all-cause mortality. J Stud Alcohol Drugs. 2016;77(2):185-98. 
27. Mukamal KJ. A safe level of alcohol consumption: the right answer demands the right question. J Intern Med. 2020;288(5):550-9.

28. Armas Rojas NB, Lacey B, Simadibrata DM, Ross S, VaronaPerez P, Burrett JA, et al. Alcohol consumption and cause-specific mortality in Cuba: prospective study of 120623 adults. EClinicalMedicine. 2021;33:100692.

29. Zhang X, Liu Y, Li S, Lichtenstein AH, Chen S, Na M, et al. Alcohol consumption and risk of cardiovascular disease, cancer and mortality: a prospective cohort study. Nutr J. 2021;20(1):13.

30. Chevli PA, Hari KJ, Kanaya AM, Talegawkar SA, Needham BL, Herrington D. Association of alcohol consumption and ideal cardiovascular health among South Asians: the mediators of atherosclerosis in South Asians living in America (MASALA) Study. Alcohol Clin Exp Res. 2020;44(9):1825-33.

31. Saito E, Inoue M, Sawada N, Charvat H, Shimazu T, Yamaji T, et al. Impact of alcohol intake and drinking patterns on mortality from all causes and major causes of death in a Japanese population. J Epidemiol. 2018;28(3):140-8.

32. Jackson CL, Hu FB, Kawachi I, Williams DR, Mukamal KJ, Rimm EB. Black-White differences in the relationship between alcohol drinking patterns and mortality among US men and women. Am J Public Health. 2015;105(Suppl 3):S534-43.

33. Wall TL, Luczak SE, Hiller-Sturmhofel S. Biology, genetics, and environment: underlying factors influencing alcohol metabolism. Alcohol Res. 2016;38(1):59-68.

34. Jorgenson E, Thai KK, Hoffmann TJ, Sakoda LC, Kvale MN, Banda $\mathrm{Y}$, et al. Genetic contributors to variation in alcohol consumption vary by race/ethnicity in a large multi-ethnic genomewide association study. Mol Psychiatry. 2017;22(9):1359-67.

35. Ma H, Li X, Zhou T, Sun D, Shai I, Heianza Y, et al. Alcohol consumption levels as compared with drinking habits in predicting all-cause mortality and cause-specific mortality in current drinkers. Mayo Clin Proc. 2021;96(7):1758-69.

36. Jani BD, McQueenie R, Nicholl BI, Field R, Hanlon P, Gallacher $\mathrm{KI}$, et al. Association between patterns of alcohol consumption (beverage type, frequency and consumption with food) and risk of adverse health outcomes: a prospective cohort study. BMC Med. 2021;19(1):8.

37. Lui CK, Kerr WC, Li L, Mulia N, Ye Y, Williams E, et al. Lifecourse drinking patterns, hypertension, and heart problems among U.S. adults. Am J Prev Med. 2020;58(3):386-95.

38 Del Bo C, Bernardi S, Marino M, Porrini M, Tucci M, Guglielmetti $S$, et al. Systematic review on polyphenol intake and health outcomes: is there sufficient evidence to define a health-promoting polyphenol-rich dietary pattern? Nutrients. 2019;11(6):1355.

39. Naimi TS, Brown DW, Brewer RD, Giles WH, Mensah G, Serdula MK, et al. Cardiovascular risk factors and confounders among nondrinking and moderate-drinking U.S. adults. Am J Prev Med. 2005;28(4):369-73.

40. Jackson R, Broad J, Connor J, Wells S. Alcohol and ischaemic heart disease: probably no free lunch. Lancet. 2005;366(9501):1911-2.

41. Fekjaer HO. Alcohol-a universal preventive agent? A critical analysis. Addiction. 2013;108(12):2051-7.

42 Shaper AG. Alcohol and mortality: a review of prospective studies. Br J Addict. 1990;85(7):837-47 discussion 49-61.

43. Andreasson S. Alcohol and J-shaped curves. Alcohol Clin Exp Res. 1998;22(7 Suppl):359S-S364.

44. Shaper AG, Wannamethee G, Walker M. Alcohol and mortality in British men: explaining the U-shaped curve. Lancet. 1988;2(8623): 1267-73.

45. Fillmore KM, Stockwell T, Chikritzhs T, Bostrom A, Kerr W. Moderate alcohol use and reduced mortality risk: systematic error in prospective studies and new hypotheses. Ann Epidemiol. 2007;17(5 Suppl):S16-23.
46. Liang W, Chikritzhs T. Reduction in alcohol consumption and health status. Addiction. 2011;106(1):75-81.

47. Newsom JT, Huguet N, McCarthy MJ, Ramage-Morin P, Kaplan MS, Bernier J, et al. Health behavior change following chronic illness in middle and later life. J Gerontol B Psychol Sci Soc Sci. 2012;67(3):279-88.

48. Power C, Rodgers B, Hope S. U-shaped relation for alcohol consumption and health in early adulthood and implications for mortality. Lancet. 1998;352(9131):877.

49. $\mathrm{Ng}$ Fat L, Shelton N. Associations between self-reported illness and non-drinking in young adults. Addiction. 2012;107(9):1612-20.

50. Ng Fat L, Cable N, Marmot MG, Shelton N. Persistent longstanding illness and non-drinking over time, implications for the use of lifetime abstainers as a control group. J Epidemiol Community Health. 2014;68(1):71-7.

51. Lemmens P, Knibbe RA, Tan F. Weekly recall and dairy estimates of alcohol consumption in a general population survey. $\mathbf{J}$ Stud Alcohol. 1988;49(2):131-5.

52. Ekholm O. Influence of the recall period on self-reported alcohol intake. Eur J Clin Nutr. 2004;58(1):60-3.

53. Dawson DA. Methodological issues in measuring alcohol use. Alcohol Res Health. 2003;27(1):18-29.

54. Duffy JC, Waterton JJ. Under-reporting of alcohol consumption in sample surveys: the effect of computer interviewing in fieldwork. Br J Addict. 1984;79(3):303-8.

55. Boniface S, Kneale J, Shelton N. Drinking pattern is more strongly associated with under-reporting of alcohol consumption than socio-demographic factors: evidence from a mixed-methods study. BMC Public Health. 2014;14:1297.

56. Stockwell T, Donath S, Cooper-Stanbury M, Chikritzhs T, Catalano P, Mateo C. Under-reporting of alcohol consumption in household surveys: a comparison of quantity-frequency, graduated-frequency and recent recall. Addiction. 2004;99(8):1024-33.

57. Latkin CA, Mai NV, Ha TV, Sripaipan T, Zelaya C, Le Minh N, et al. Social desirability response bias and other factors that may influence self-reports of substance use and HIV risk behaviors: a qualitative study of drug users in Vietnam. AIDS Educ Prev. 2016;28(5):417-25.

58. van de Luitgaarden IAT, Beulens JWJ, Schrieks IC, Kieneker LM, Touw DJ, van Ballegooijen AJ, et al. Urinary ethyl glucuronide can be used as a biomarker of habitual alcohol consumption in the general population. J Nutr. 2019;149(12):2199-205.

59.• Brien SE, Ronksley PE, Turner BJ, Mukamal KJ, Ghali WA. Effect of alcohol consumption on biological markers associated with risk of coronary heart disease: systematic review and meta-analysis of interventional studies. BMJ. 2011;342:d636. This systematic review and meta-analysis of interventional studies provides indirect pathophysiological support for a protective effect of moderate alcohol use on coronary heart disease by investigating cardiovascular biomarkers

60. Huang Y, Li Y, Zheng S, Yang X, Wang T, Zeng J. Moderate alcohol consumption and atherosclerosis : Meta-analysis of effects on lipids and inflammation. Wien Klin Wochenschr. 2017;129(21-22):835-43.

61. Schrieks IC, Heil AL, Hendriks HF, Mukamal KJ, Beulens JW. The effect of alcohol consumption on insulin sensitivity and glycemic status: a systematic review and meta-analysis of intervention studies. Diabetes Care. 2015;38(4):723-32.

62. Roerecke M, Kaczorowski J, Tobe SW, Gmel G, Hasan OSM, Rehm J. The effect of a reduction in alcohol consumption on blood pressure: a systematic review and meta-analysis. Lancet Public Health. 2017;2(2):e108-20. 
63. Hirst JA, Aronson JK, Feakins BG, Ma C, Farmer AJ, Stevens RJ. Short- and medium-term effects of light to moderate alcohol intake on glycaemic control in diabetes mellitus: a systematic review and meta-analysis of randomized trials. Diabet Med. 2017;34(5):604-11.

64. Shai I, Wainstein J, Harman-Boehm I, Raz I, Fraser D, Rudich A, et al. Glycemic effects of moderate alcohol intake among patients with type 2 diabetes: a multicenter, randomized, clinical intervention trial. Diabetes Care. 2007;30(12):3011-6.

65 DeJong W. The Moderate Alcohol and Cardiovascular Health Trial: public health advocates should support good science, not undermine it. Eur J Prev Cardiol. 2020;28(15):e22-4.

66. Bennett DA, Holmes MV. Mendelian randomisation in cardiovascular research: an introduction for clinicians. Heart. 2017;103(18):1400-7.

67. van de Luitgaarden IAT, van Oort S, Bouman EJ, Schoonmade LJ, Schrieks IC, Grobbee DE, et al. Alcohol consumption in relation to cardiovascular diseases and mortality: a systematic review of Mendelian randomization studies. Eur J Epidemiol. 2021. This systematic review summarizes the current evidence of moderate alcohol consumption on CVD from MR studies.

68. Burgess S, Thompson S. Mendelian randomization: methods for using genetic variants in causal estimation. Boca Raton: Chapman and Hall/CRC; 2015.

69 Burgess S, Davies NM, Thompson SG. Consortium EP-I. Instrumental variable analysis with a nonlinear exposure-outcome relationship. Epidemiology. 2014;25(6):877-85.

70. Peng M, Zhang J, Zeng T, Hu X, Min J, Tian S, et al. Alcohol consumption and diabetes risk in a Chinese population: a Mendelian randomization analysis. Addiction. 2019;114(3):436-49.

71. Silverwood RJ, Holmes MV, Dale CE, Lawlor DA, Whittaker JC, Smith GD, et al. Testing for non-linear causal effects using a binary genotype in a Mendelian randomization study: application to alcohol and cardiovascular traits. Int J Epidemiol. 2014;43(6):1781-90.

72 Mukamal KJ, Stampfer MJ, Rimm EB. Genetic instrumental variable analysis: time to call mendelian randomization what it is. The example of alcohol and cardiovascular disease. Eur $\mathbf{J}$ Epidemiol. 2020;35(2):93-7.

73. Kranzler HR, Zhou H, Kember RL, Vickers Smith R, Justice AC, Damrauer $\mathrm{S}$, et al. Genome-wide association study of alcohol consumption and use disorder in 274,424 individuals from multiple populations. Nat Commun. 2019;10(1):1499.

74. Brumpton B, Sanderson E, Heilbron K, Hartwig FP, Harrison S, Vie GA, et al. Avoiding dynastic, assortative mating, and population stratification biases in Mendelian randomization through within-family analyses. Nat Commun. 2020;11(1):3519.

75. Howe LJ, Lawson DJ, Davies NM, St Pourcain B, Lewis SJ, Davey Smith G, et al. Genetic evidence for assortative mating on alcohol consumption in the UK Biobank. Nat Commun. 2019;10(1):5039.

76 Felitti VJ, Anda RF, Nordenberg D, Williamson DF, Spitz AM, Edwards V, et al. Relationship of childhood abuse and household dysfunction to many of the leading causes of death in adults. The Adverse Childhood Experiences (ACE) Study. Am J Prev Med. 1998;14(4):245-58.

77. Patsopoulos NA. A pragmatic view on pragmatic trials. Dialogues Clin Neurosci. 2011;13(2):217-24.

78. Ford I, Norrie J. Pragmatic trials. N Engl J Med. 2016;375(5):454-63.

79. Grobbee DE, Hoes A. Clinical epidemiology: principles, methods, and applications for clinical research. Burlinton: Jones \& Bartlett Publishers; 2014.

80. Hudson J, Fielding S, Ramsay CR. Methodology and reporting characteristics of studies using interrupted time series design in healthcare. BMC Med Res Methodol. 2019;19(1):137.

81. Bernal JL, Cummins S, Gasparrini A. Interrupted time series regression for the evaluation of public health interventions: a tutorial. Int J Epidemiol. 2016;46(1):348-55.

82. Kalinowski A, Humphreys K. Governmental standard drink definitions and low-risk alcohol consumption guidelines in 37 countries. Addiction. 2016;111(7):1293-8.

83. Stockwell T, Room R. Constructing and responding to low-risk drinking guidelines: conceptualisation, evidence and reception. Drug Alcohol Rev. 2012;31(2):121-5.

84. Lawlor DA, Tilling K, Davey SG. Triangulation in aetiological epidemiology. Int J Epidemiol. 2016;45(6):1866-86.

85. Munafò MR, Davey SG. Robust research needs many lines of evidence. Nature. 2018;553(7689):399-401.

86. Organization WH. Global status report on alcohol and health 2018 Geneva. Licence: CC BY-NC-SA 30 IGO2018.

Publisher's Note Springer Nature remains neutral with regard to jurisdictional claims in published maps and institutional affiliations. 